\title{
Maintenance of a large pericentric inversion generated by the hobo transposable element in a transgenic line of Drosophila melanogaster
}

\author{
S Aulard ${ }^{1}$, P Vaudin ${ }^{2}$, V Ladevèze ${ }^{3}$, N Chaminade ${ }^{1}$, G Périquet ${ }^{2}$ and F Lemeunier $^{1}$ \\ ${ }^{1}$ Laboratoire Populations, Génétique et Evolution, Centre National de la Recherche Scientifique, Avenue de la Terrasse, 91198 Gif-sur- \\ Yvette Cedex, France; 'Institut de Recherche sur la Biologie de l'Insecte, Université François Rabelais, UFR Sciences et Techniques, Parc \\ Grandmont, 37200 Tours, France; ${ }^{3}$ Laboratoire de Génétique Cellulaire et Moléculaire, Université de Poitiers, CHU, 86021 Poitiers \\ Cedex, France
}

\begin{abstract}
The impact of the hobo transposable element in the global reorganization of the Drosophila melanogaster genome has been investigated in transgenic lines generated by the injection of hobo elements into the Hikone strain, which lacked them previously. Extensive surveys of transgenic lines followed for 250 generations have identified 13 inversions with hobo inserts at most breakpoints. One of these inversions is pericentric on chromosome 2. It has been maintained in the line where it was discovered and in several
\end{abstract}

sublines at frequencies from 0.19 to 0.45 , generating stable chromosomal polymorphisms, similar to cosmopolitan paracentric inversions in natural populations. Individuals homozygous for this inversion were viable and fertile, allowing the creation of a new homozygous strain. Hobo elements were still active after 250 generations.

Heredity (2004) 92, 151-155, advance online publication, 7 January 2004; doi:10.1038/sj.hdy.6800375

Keywords: Drosophila melanogaster, hobo transposable element; polymorphic pericentric inversion; in situ hybridization

\section{Introduction}

Among the mobile elements in Drosophila melanogaster, three independent systems ( $P, I$ and hobo) produce aberrant germline events, such as high mutation rate, infertility and elevated levels of chromosome instability (Blackman and Gelbart, 1989). In recent years, transposable elements have been suggested to play an important role in genome evolution, as major players in genomic restructuring (McDonald, 1998). The contribution of hobo elements to chromosome instability was demonstrated by Lim and co-workers in several studies (Lim, 1988; Ho et al, 1993; Sheen et al, 1993; Eggleston et al, 1996). In order to trace the fate of hobo elements after their injection into a Hikone strain devoid of these elements, seven transgenic lines were followed over 250 generations (Ladevèze et al, 1994; Galindo et al, 1995; Ladevèze et al, 1998a, b, 2001). Hobo elements increased in each line, showing the autonomous amplification by transposition of this element. At the chromosomal level, hobo elements appeared to be actively involved in the formation of rearrangements. In all, 13 new inversions were observed among the different lines, most of them with a hobo insert at one or both inversion breakpoints. In the present study, we focus on a long-time survey of one of these lines (no. 11), in which a large hobo-mediated pericentric inversion on chromosome 2 was maintained after more than 120 unselected generations. The consequences of such a polymorphism on chromosomal restructuring of the Drosophila genome are discussed.

\section{Materials and methods}

\section{Basic strains and microinjection}

Drosophila melanogaster embryos were transformed with pHFL1 using the standard microinjection technique (Spradling and Rubin, 1982). The microinjected plasmid pHFL1 (Blackman et al, 1989) contains one autonomous hobo element and some adjacent genomic DNA from cytogenetic locus $94 E$, cloned in the pBLUESCRIPT KS plasmid. The strain used as a receptor was Hikone, an E-type strain in the hobo system that lacks hobo elements. It was also of the $\mathrm{R}$ and $\mathrm{M}$ types, that is, devoid of $I$ and $P$ transposable elements. In all, 30 independent lines were found by individually crossing one injected fly with one noninjected Hikone fly (Ladevèze et al, 1994). Subsequently, each line was bred en masse. Lines were analysed at generation 2 using the Southern blot technique to check for the presence of hobo elements. Seven hobopositive lines were obtained. Each was maintained independently by mass matings (ca. 100 individuals) at $25^{\circ} \mathrm{C}$ in the following generations. Line 11, in which the large pericentric inversion was found, was duplicated at generation 145 .

\section{In situ hybridization}

In every 10-15 generations, 10 larvae were screened for the presence of hobo element on their chromosomes in the

replicates of line 11. In situ hybridization of biotinylated
Correspondence: S Aulard, Laboratoire Populations, Génétique et Evolu91198 Gif-sur-Yvette Cedex, France.

E-mail : Sylvie.Aulard@pge.cnrs-gif.fr

Received 27 November 2002; accepted 10 June 2003 
probes (Boehringer kit) to salivary gland polytene chromosomes was adapted from Lemeunier and Aulard (2000). The pHFL1 plasmid used in the microinjection step was used as a probe for the in situ hybridizations. Under our experimental conditions, no hybridization site, including the $94 E$ locus, was observed when this pHFL1 probe was used on the parental Hikone strain chromosomes.

\section{Drosophila stocks used in the analyses}

All mutations and balancer chromosomes are described in Lindsley and Zimm (1992).

$C y^{O} / T(2-3)$ : second chromosome balanced strain $\operatorname{In}(2-$ LR)O, dp ${ }^{\mathrm{lvl}}, \mathrm{Cy}, \mathrm{pr}, \mathrm{cn}^{2} / \mathrm{T}(2-3) \mathrm{wg}^{-}, \mathrm{cn}, \mathrm{bw}$.

$d p, b, c n:$ a strain homozygous for the second chromosome mutations dumpy (dp 2-13.0), black (b 248.5) and cinnabar (cn 2-57.5).

\section{Isolation of the $\ln (2 \mathrm{LR})$ chromosome}

$\operatorname{In}(2 L R) 27 B ; 53 C$ : the large hobo-mediated pericentric inversion on the second chromosome, observed in this study and denoted simply as $\operatorname{In}(2 L R)$ in the rest of this paper.

In order to know if adults homozygous for the $\operatorname{In}(2 L R)$ chromosome are viable and fertile, crosses have been performed involving 30 randomly chosen males from line 11 and $30 \mathrm{dp}, b, \mathrm{cn}$ females. In all, 61 F1 females were then individually testcrossed with three $d p, b, c n$ males. The progeny of the 61 females were scored for the presence or absence of recombinants between the $b$ and $c n$ loci. As $\operatorname{In}(2 L R)$ spans the $b-c n$ interval, the absence of recombinant progeny indicated that the maternal F1 female was heterozygous for the $\operatorname{In}(2 L R)$ chromosome. Six progeny of this type were observed with one half of genotypes $\operatorname{In}(2 L R) / d p, b, c n$. From each one of these progeny, a single $\left[\mathrm{dp}^{+} b^{+} \mathrm{cn} n^{+}\right]$male was crossed with three $C y^{O / T}(2-3)$ females to isolate the $\operatorname{In}(2 L R) / C y^{O}, d p, c n$ adults in their descendants. These individuals were then crossed together and their progeny analysed to look for the presence of wild-type adults, corresponding to homozygous $\operatorname{In}(2 L R) / \operatorname{In}(2 L R)$ if they were viable.

\section{Genetics and molecular tests}

The hobo status of line 11 was measured by crossing the line with Hikone and $23.5^{*} / \mathrm{Cy}$ and looking for the level of induced gonadal dysgenesis (\% GD) in order to estimate the levels of hobo-activity and repression potential (Yannopoulos et al, 1987; Ladevèze et al, 1994). Standard techniques were used for DNA extraction, gel electrophoresis, blotting and hybridization (Maniatis et al, 1982). Genomic DNA of adult flies was digested by XhoI, which cuts near each end of the hobo sequence, yielding a $2.6 \mathrm{~kb}$ fragment from complete hobo elements. After electrophoresis on a $1 \%$ agarose gel, transfer and hybridization were performed on Nytran membranes (Schleicher and Schuell). The probe used was the $2.6 \mathrm{~kb}$ fragment generated by XhoI digestion of pHFL1. Hybridization was carried out overnight at $65^{\circ} \mathrm{C}$ in $0.25 \mathrm{M}$ phosphate buffer $\mathrm{pH} 7.2,1 \mathrm{mM}$ ethylene diaminetetraacetate, $7 \%$ sodium dodecyl sulphate. The membranes were washed at $65^{\circ} \mathrm{C}$ for $20 \mathrm{~min}$ in $2 \times$ standard saline citrate (SSC), $20 \mathrm{~min}$ in $1 \times$ SSC and $20 \mathrm{~min}$ in $0.5 \times$ SSC. Filters were then exposed to an X-ray film for 1 or 2 days.

\section{Results}

Observation of a pericentric inversion and its fate in the evolving population

At generation 130, we observed one individual heterozygous for a large pericentric inversion on the second chromosome. Breakpoints were at $27 B$ on the left arm and $53 \mathrm{C}$ on the right arm of the chromosome (Figure 1). Hobo insertions were detected at both breakpoints. The frequency of the $\operatorname{In}(2 L R)$ chromosome was a few percent as only one inverted chromosome was found among the 20 analysed.

As this inversion was also observed at generations 142 and 145, a duplication of line 11 was made at this last generation. The two sublines were maintained en masse independently under the same conditions and analysed during the following 100 generations. Figure 2 shows the maintenance of this chromosomal polymorphism during that time, with an average frequency of 0.28 and 0.29 for the $\operatorname{In}(2 L R)$ chromosome in the two sublines.

In all, 14 isofemale sublines were initiated from line 11 at generation 145. Cytological analysis of their progeny identified four sublines in which $\operatorname{In}(2 L R)$ was detected. These four sublines were maintained independently,
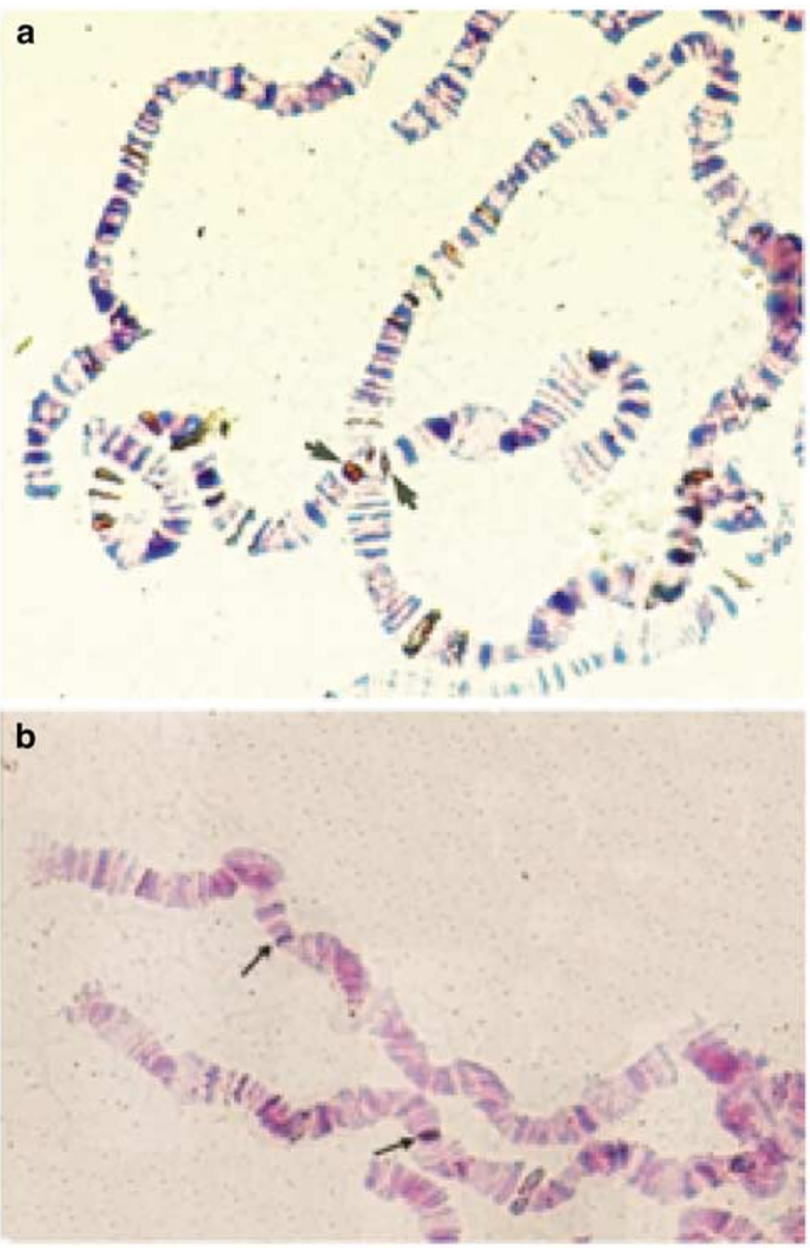

Figure 1 In situ hybridization with the biotinylated pHFL1 probe to inverted chromosome. (a) Heterokaryotype $\operatorname{In}(2 L R) 27 B-53 C / S t$. (b) Homokaryotype $\operatorname{In}(2 L R) 27 B-53 C / \operatorname{In}(2 L R) 27 B-53 C$. Arrows point to hobo inserts at the chromosomal breakpoints. 

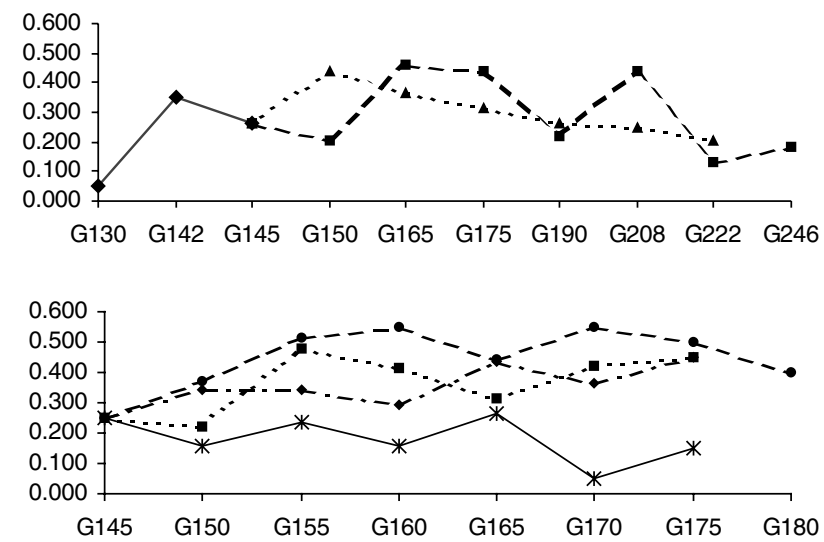

Figure 2 Maintenance of the pericentric inverted chromosome. Top: Two subpopulations founded by a duplication en masse of the original line 11. Bottom: Four subpopulations each founded by one inseminated female from the original line 11.

en masse, and analysed over 30-35 generations. The $\operatorname{In}(2 L R)$ frequency was estimated to be 0.25 initially because it is most probable that only one of the two original parents was heterozygous, assuming the female mated only one time. Indeed, the first homozygous larva in the parental line 11 was detected at generation 150. Figure 2 shows the maintenance of the chromosomal polymorphism, with $\operatorname{In}(2 L R)$ average frequencies from 0.19 to 0.45 .

\section{Viability and fertility of $\operatorname{In}(2 L R)$ homozygous adults}

The finding of larvae homozygous for $\operatorname{In}(2 L R)$, in line 11 and in the four isofemale sublines, demonstrates the viability of this genotype, at least at the third instar. In order to determine if homozygous adults are viable and fertile, appropriate crosses were performed (see Materials and methods). Each cross between females and $\operatorname{In}(2 L R) / C y^{\circ}, d p, c n$ males gave abundant progeny (ie 200 offspring per female over 3-4 days). Among progeny, two types of adults were observed: $2 / 3$ of [Cy] phenotype corresponding to $\operatorname{In}(2 L R) / C y^{\circ}, d p, c n$ individuals and $1 / 3$ of [wild type] corresponding to $\operatorname{In}(2 L R) /$ $\operatorname{In}(2 L R)$. These results show that the $\operatorname{In}(2 L R)$ chromosome is equally viable in the homozygous and the heterozygous states. Moreover, the homozygous individuals were fertile when intercrossed, producing abundant progeny and homozygous $\operatorname{In}(2 L R)$ lines. Their homozygous karyotypes (Figure 1) were checked by observation of salivary gland polytene chromosomes.

\section{Genetic and molecular analyses}

The genetic tests show that from generation 130 onwards, line 11 has not acquired a strong hobo-activity potential (with values around 5-10\% of the induced GD sterility) and has a low repression potential for hobo activity (79-86\% of the induced GD sterility).

Southern blot analyses of line 11, from generation 63 onwards, show the presence of the $2.6 \mathrm{~kb}$ XhoI internal fragment expected for complete hobo HFL1 elements (Figure 3). Smaller restriction fragments that might have originated from internal deletions were observed after G63. Some bands were observed corresponding to deleted elements, but they were always very rare and none became fixed.

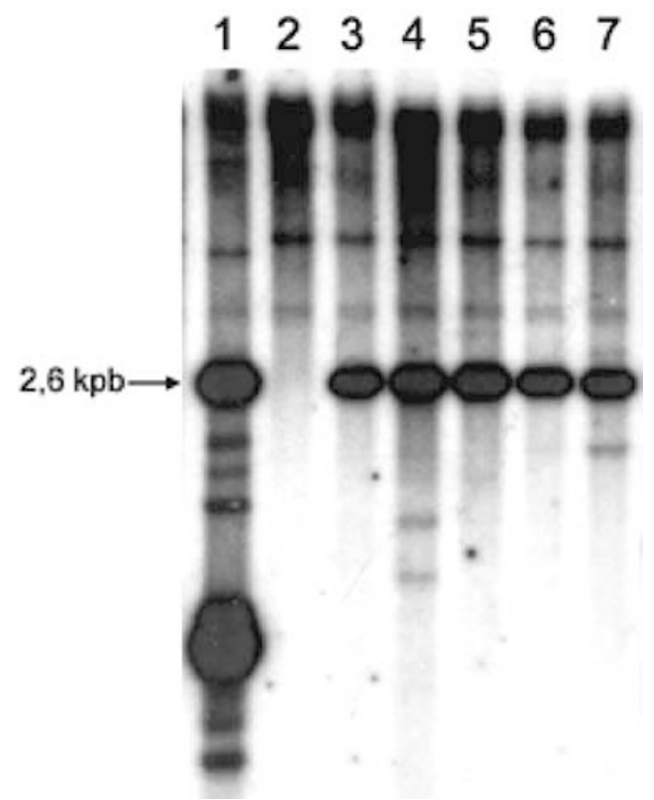

Figure 3 Southern blot analysis of hobo sequences in line 11 at different generations. The probe used was the $2.6 \mathrm{~kb}$ fragment generated by XhoI digestion of pHFL1. Lane 1 corresponds to Tours82 strain as a positive control, lane 2 to Hikone strain as negative control, lanes 3-7 to line 11 at G63, G71, G110, G125 and G208, respectively.

\section{Distribution of hobo inserts}

At G8, an average of 1.7 hobo inserts per individual was detected by in situ hybridization in line 11. The number of insertion sites increased slowly until G81 (seven inserts). At G130, this number was three times higher (21) and increased regularly during the 80 following generations. By generation 208, the number reached 31 . However, the increase in inserts was not similar for all chromosomes. Since G130, we observed that the number of insertion sites was always higher on both arms of chromosome 2 compared to chromosomes $X$ and 3 (Figure 4). The total number of hobo inserts detected during these 208 generations is 20 for the $X, 77$ and 87 for the left and right arms of chromosome 2, respectively, and 37 and 36 for those of chromosome 3 . As in the other transgenic lines, no hybridization was observed on chromosome 4 or in the chromocentre.

\section{Discussion}

From the seven transgenic lines obtained, 13 chromosomal inversions were observed (Ladevèze et al, 1994, 1998a, $b$ and unpublished data): nine paracentric (two on the $X$, one on the second and six on the third chromosomes) and four pericentric (two on each of the large autosomes). Most were detected in a single larva. Two paracentric inversions on $3 L$ were observed twice, at different generations. The pericentric inversion $\operatorname{In}(2 L R) 27 B ; 53 C$ is the only one retained for many generations. Over about 120 generations, it was present at stable frequencies (ca. 0.28-0.29) in the different subpopulations, showing the unselected maintenance of a chromosomal polymorphism generated by hobo elements.

Heterozygotes for pericentric inversions are expected to be semisterile because recombination within the 


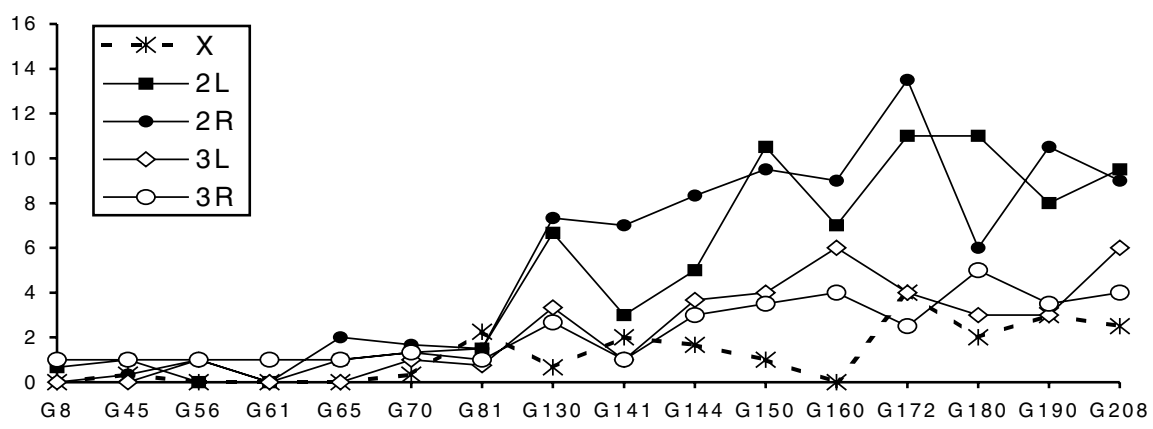

Figure 4 Mean number of insertion sites per individual, for each chromosome, at different generations.

inverted region produces aneuploid gametes. Owing to this underdominance, these arrangements should quickly be eliminated from populations when they first arise. This would explain the quasi-absence of polymorphism for this kind of inversion in nature. The only example of such a polymorphism in D. melanogaster was recorded by Aulard (1990), Coyne et al (1991) and Aulard et al (2002). Coyne et al (1993) found that fertility was not reduced in many cases, for laboratory-induced pericentric inversions, due to suppressed crossingover in heterokaryotypic individuals. This lack of underdominance depends on the position of breakpoints on the chromosome. These 'sensitive sites', described on chromosomes 2 and 3, appear to reduce recombination in a heterozygous inversion whose breakpoints are nearby. However, these sites do not correspond to our $\operatorname{In}(2 L R)$ breakpoints.

The maintenance of the $\operatorname{In}(2 L R)$ may result either from a selection effect (for example, an advantage of the heterozygote) or from a stochastic event (a transient phase before extinction or fixation by genetic drift). As the size of the experimental populations is always limited, the role of genetic drift must be taken into consideration. The question is whether maintenance over 120 generations is very probable in our experimental populations. Kimura (1971) showed that for a population of size $N$, the probability for a neutral nonrecurrent mutation to be lost is $(1-1 / 2 N)$. In our case, $N$ was about 100 and probability of loss of the inversion was about 0.995 . Moreover, taking $t_{\mathrm{o}}$ as the number of generations until loss, Kimura shows that its mean is given by: $2\left(N_{\mathrm{e}} / N\right) \log _{\mathrm{e}}(2 N)$ and its variance by: $\left(16 N_{\mathrm{e}}^{2} / N-\left(2 N_{\mathrm{e}} / N\right) \log _{\mathrm{e}} 2 N\right)^{2}$. If we consider that the effective size $\left(N_{\mathrm{e}}\right)$ of Drosophila populations is about $0.7 N$ (Crow and Morton, 1955), we obtain a mean value of 7.4 generations (with a standard deviation of 27.0) for the time to loss of a chromosomal inversion if neutral. Therefore, in more than $99 \%$ of such cases, a neutral inversion will not persist more than 5060 generations. Compared with the 120 generations of maintenance observed for the $\operatorname{In}(2 L R)$ chromosome in both subpopulations and the $4 \times 40$ generations in the four isofemale replicates, our results reject the genetic drift hypothesis and point to a very likely role of selection in the maintenance of this chromosomal rearrangement.

In this case, a possible explanation resides in the advantage of the chromosomal heterozygote $\operatorname{In}(2 L R) /$ Standard. Simulation analyses fitting the model of selection with overdominance were performed on our observed data. They allowed us to estimate the selective values of each genotype. The fitness estimates $w_{1}=0.94$ for $S t d / S t d, w_{2}=1$ for $\operatorname{In}(2 L R) / S t d$ and $w_{3}=0.85$ for $\operatorname{In}(2 L R) / \operatorname{In}(2 L R)$ are compatible with a stable polymorphism and an equilibrium frequency of 0.28 for $\operatorname{In}(2 L R)$, as observed in our experiments. The frequency of the $\operatorname{In}(2 L R)$ chromosome will increase from 0.05 (initial estimate at its first observation) to around 0.25 in about 30-40 generations, in a way similar to our experimental populations.

At the evolutionary level, such an inversion could be maintained for many generations, producing a chromosomal polymorphism similar to the ones encountered in natural populations for paracentric cosmopolitan inversions of $D$. melanogaster (review in Lemeunier and Aulard, 1992) and many other Drosophila species (review in Sperlich and Pfriem, 1986). Moreover, that the $\operatorname{In}(2 L R)$ inversion is viable and fertile shows that a hobo element can induce inversions that become fixed in small populations leading to new homokaryotypic populations.

In accordance with these results, several observations suggest a possible impact of hobo element in the generation of chromosomal inversions in natural populations. In a natural population of $D$. melanogaster in the Hawaiian islands, four endemic inversions, with hobo elements at one or both breakpoints, have been described, but the cosmopolitan inversions on the same chromosome do not show this association (Lyttle and Haymer, 1992). One of the individuals in this population carried an unstable second chromosome, as revealed by laboratory backcrosses, with 12 hobo elements. About 1\% of the progeny of this fly had new inversions, duplications or transpositions in the second chromosome. Many of the breakpoints of these rearrangements were sites occupied by hobo elements in a way similar to those generated in our experimental populations (Ladevèze et al, 1998a).

In a Greek natural population of D. melanogaster, an analysis extending over three seasons showed that three out of the five cosmopolitan inversions analysed and two of the three endemics were found to have hobo inserts at or very near one of the two breakpoints. A comparative analysis of the chromosomal distribution of the $P$ element demonstrated that two out of the five cosmopolitan inversions and one of the three endemics had a $P$ insertion at or very near one of the two breakpoints. None of the inversions detected had $P$ or hobo insertions at both breakpoints (Zabalou et al, 1994).

Inversions in which the breakpoints lack hobo elements may represent cases in which hobo excised after causing 
the rearrangement, or may have deleted copies too small to be detected by in situ hybridization. Another explanation is that some inversions are generated by transposable elements other than hobo or by agents other than transposable elements. However, in line 11, 11 other transposable elements (Bel, copia, DocA, gypsy, mdg1, mdg3, $P$, roo, Stalker, 412, 1731) have been regularly checked by in situ hybridization and in no cases have been found at an inversion breakpoint. This points to a major role of hobo in our experiments due to their introduction as active elements in the initial strain, but does not rule out the role of other elements in other lines (Lim and Simmons, 1994). The hobo elements were still active after 250 generations. No GD was observed in the Hikone strain, devoid of hobo elements. The GD status of the transgenic lines showed a global evolution towards a low hobo-activity potential with a variable level of repression potential. Southern blots revealed the presence of many full-length elements, the activity of which was confirmed by continued mobilization after 250 generations. Many new sites of transposition were observed until the last generations, showing that the hobo element can always participate in the genesis of genetic variability (genic and chromosomal).

These observations show that hobo elements are actively involved in the formation of rearrangements that generate important chromosomal polymorphisms. In nature, we can therefore consider the transposable elements as a reservoir of mutagenic agents, which help to reshape the structure of the Drosophila genome.

\section{Acknowledgements}

We thank C Barjolin for technical assistance. We are most grateful to $\mathrm{J}$ Brookfield and anonymous reviewers for helpful suggestions. This work was supported by a grant from the programme GDR 2157 'Evolution des Eléments Transposables: du Génome aux Populations', Centre National de la Recherche Scientifique.

\section{References}

Aulard S (1990). Polymorphisme chromosomique de Drosophila melanogaster, en Afrique et dans les îles de l'Océan Indien. Doctoral Thesis, University of Paris (P6): France.

Aulard S, David JR, Lemeunier F (2002). Chromosomal inversion polymorphism in Afrotropical populations of Drosophila melanogaster. Genet Res Camb 79: 49-63.

Blackman RK, Gelbart WM (1989). The transposable element hobo of Drosophila melanogaster. In: Berg DE, Howe MM (eds) Mobile DNA. American Society for Microbiology: New York pp 523-529.

Blackman RK, Macy M, Koehler MD, Grimaila R, Gelbart WM (1989). Identification of a fully-functional hobo transposable element and its use for germ-line transformation of Drosophila. EMBO J 8: 211-217.

Coyne JA, Aulard S, Berry A (1991). Lack of underdominance in a naturally occurring pericentric inversion in Drosophila melanogaster and its implications for chromosome evolution. Genetics 129: 791-802.

Coyne JA, Meyers W, Crittenden AP, Sniegowski P (1993). The fertility effects of pericentric inversions in Drosophila melanogaster. Genetics 134: 487-496.

Crow JF, Morton NE (1955). Measurement of gene frequency drift in small populations. Evolution 9: 202-214.
Eggleston WB, Rim NR, Lim JK (1996). Molecular characterization of hobo-mediated inversions in Drosophila melanogaster. Genetics 144: 647-656.

Galindo MI, Ladevèze V, Lemeunier F, Kalmes R, Périquet G, Pascual L (1995). Spread of the autonomous transposable element hobo in the genome of Drosophila melanogaster. Mol Biol Evol 12: 723-734.

Ho YT, Weber SM, Lim JK (1993). Interacting hobo transposons in an inbred strain and interaction regulation in hybrids of Drosophila melanogaster. Genetics 134: 895-908.

Kimura M (1971). The Neutral Theory of Molecular Evolution. Cambridge University Press: Cambridge.

Ladevèze V, Aulard S, Chaminade N, Biémont C, Périquet G, Lemeunier F (2001). Dynamics of the hobo transposable element in transgenic lines of Drosophila melanogaster. Genet Res Camb 77: 135-142.

Ladevèze $\mathrm{V}$, Aulard $\mathrm{S}$, Chaminade $\mathrm{N}$, Périquet $\mathrm{G}$, Lemeunier $\mathrm{F}$ (1998a). Hobo transposons causing chromosomal breakpoints. Proc R Soc London Ser B 265: 1157-1159.

Ladevèze V, Chaminade N, Périquet G, Lemeunier F (1998b). Transmission pattern of hobo transposable element in transgenic lines of Drosophila melanogaster. Genet Res Camb 71: 97-107.

Ladevèze V, Galindo MI, Pascual L, Périquet G, Lemeunier F (1994). Invasion of the hobo transposable element studied by in situ hybridization on polytene chromosomes of Drosophila melanogaster. Genetica 93: 91-100.

Lemeunier F, Aulard S (1992). Inversion polymorphism in Drosophila melanogaster. In: Krimbas CB, Powell JR (eds) Drosophila Inversion Polymorphism. CRC Press: Boca Raton, FL pp 339-405.

Lemeunier F, Aulard S (2000). Drosophila chromosome study techniques. In: Popescu P, Hayes H, Dutrillaux B (eds) Techniques in Animal Cytogenetics. Springer: Berlin, Heidelberg and INRA Paris pp 137-149.

Lim JK (1988). Intrachromosomal rearrangements mediated by hobo transposons in Drosophila melanogaster. Proc Natl Acad Sci USA 85: 9153-9157.

Lim JK, Simmons MJ (1994). Gross chromosome rearrangements mediated by transposable elements in Drosophila melanogaster. BioEssays 16: 269-275.

Lindsley D, Zimm G (1992). The Genome of Drosophila melanogaster. Academic Press: San Diego.

Lyttle TW, Haymer DS (1992). The role of the transposable element hobo in the origin of endemic inversions in wild populations of Drosophila melanogaster. Genetica 86: 113-126.

Maniatis T, Fritsh EF, Sambrook J (1982). Molecular Cloning. A Laboratory Manual. Cold Spring Harbor Laboratory Press: Cold Spring Harbor, NY.

McDonald JF (1998). Transposable elements, gene silencing and macroevolution. Trends Ecol Evol 13: 94-95.

Sheen FM, Lim JK, Simmons MJ (1993). Genetic instability in Drosophila melanogaster mediated by hobo transposable elements. Genetics 133: 315-334.

Sperlich D, Pfriem P (1986). Chromosomal polymorphism in natural and experimental populations. In: Ashburner $M$, Carson HL, Thompson Jr JN (eds) The Genetics and Biology of Drosophila. Academic Press: New York Vol 3e, pp 257-309.

Spradling AC, Rubin GM (1982). Transposition of cloned $P$ elements into Drosophila germ line chromosomes. Science 218: 341-347.

Yannopoulos G, Stamatis N, Monastirioti M, Hatzopoulos P, Louis C (1987). Hobo is responsible for the induction of hybrid dysgenesis by strains of Drosophila melanogaster bearing the male recombination factor 23.5MRF. Cell 49: 487-495.

Zabalou S, Alahiotis SN, Yannopoulos G (1994). A three-season comparative analysis of the chromosomal distribution of $P$ and hobo mobile elements in a natural population of Drosophila melanogaster. Hereditas 120: 127-140. 